J. DIFFERENTIAL GEOMETRY

82 (2009) 101-114

\title{
$k$-NORMAL SURFACES
}

\author{
Evgeny Fominykh \& Bruno Martelli
}

\begin{abstract}
Following Matveev, a k-normal surface in a triangulated 3manifold is a generalization of both normal and (octagonal) almost normal surfaces. Using spines, complexity, and Turaev-Viro invariants of 3-manifolds, we prove the following results:

- a minimal triangulation of a closed irreducible or a bounded hyperbolic 3-manifold contains no non-trivial $k$-normal sphere;

- every triangulation of a closed manifold with at least 2 tetrahedra contains some non-trivial normal surface;

- every manifold with boundary has only finitely many triangulations without non-trivial normal surfaces.

Here, triangulations of bounded manifolds are actually ideal triangulations. We also calculate the number of normal surfaces of nonnegative Euler characteristics which are contained in the conjecturally minimal triangulations of all lens spaces $L_{p, q}$.
\end{abstract}

\section{Introduction}

We study in this paper the existence and number of (generalizations of) normal surfaces in various triangulated 3-manifolds. Theorem 1 below concerns the existence of (a generalization of) normal spheres in minimal triangulations. Theorems 2 and 3 then show that, with finitely many exceptions, all triangulations of a given manifold contain nontrivial normal surfaces. Finally, Theorem 5 calculates the number of normal surfaces of genus 0 and 1 in a family of triangulated lens spaces.

Normal surfaces. A normal surface in a triangulated 3-manifold is a surface intersecting each tetrahedron in triangles and squares, as in Fig. 1-(1). Since every incompressible surface (in an irreducible manifold) can be isotoped into normal position, normal surfaces can be used to investigate the set of incompressible surfaces algorithmically.

Both authors are partially supported by the INTAS project "CalcoMet-GT" 0351-3663. The first author is partially supported by the Russian Foundation for Basic Research (project 07-01-96026) and the Integration Grant of the Ural — Siberian Divisions of the Russian Academy of Sciences.

Received 05/05/2006. 

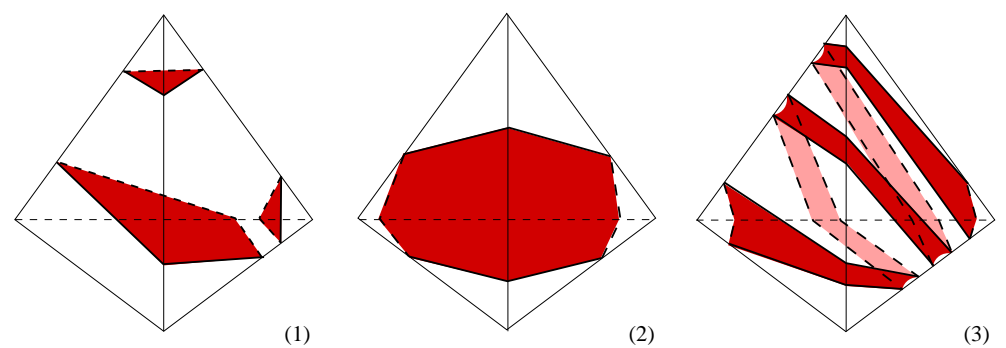

Figure 1. Some normal discs in a $k$-normal surface. In (3) we may wind the disc arbitrarily many times, getting discs with arbitrarily many intersections with the edges of the tetrahedron.

An almost normal surface is usually defined by admitting also finitely many more types of intersections with some tetrahedra, such as for instance the octagon of Fig. 1-(2). By a beautiful result of Rubinstein and Thompson [11], almost normal surfaces can be used to find algorithmically some important types of compressible surfaces, such as a 2 -sphere in $S^{3}$.

Matveev defined in [7] a $k$-normal surface as a surface that intersects each tetrahedron in polygons, having consecutive vertices in distinct edges of the tetrahedron, and incident to each such edge at most $k$ times. With this definition, a normal surface is just 1-normal, and an almost normal surface with octagons is 2-normal. Polygons may have arbitrarily many edges as $k$ increases, as suggested by Fig. 1-(3).

A surface which is $k$-normal for some $k$ was also called spinal by Frohman and Kania-Bartoszynska in [4]. We prove here the following.

Theorem 1. Let $M$ be a closed irreducible or a bounded hyperbolic 3-manifold, different from $S^{3}, \mathbb{R P}^{3}$, and $L_{3,1}$. A minimal triangulation of $M$ does not contain any non-trivial $k$-normal sphere, for all $k$.

When the manifold is bounded here we actually talk about ideal triangulations. A $k$-normal surface is trivial when it is made only of triangles. See Section 1 for definitions. Theorem 1 for normal spheres was known to Matveev in 1990, and its proof is essentially contained in [6, Theorem B] as a byproduct of his complexity theory. It was then extended to the 2-normal case by Jaco and Rubinstein [5], see [7] for a short proof. We prove it in the general case using spines. It turns out that $k$-normal surfaces are sometimes better studied with spines rather than with triangulations: the main reason for that is that by cutting a spine along a $k$-normal surface we get another spine, while by cutting a triangulation we get a polyhedral subdivision which is never a triangulation.

Existence of normal surfaces. A connection between normal surfaces and Turaev-Viro invariants was noted in [4]. We use here a nice version 
of the homology-free 5th Turaev-Viro invariant, called the $t$-invariant, due to Matveev, Ovchinnikov, and Sokolov [9], to prove the following results.

Theorem 2. Every compact 3-manifold has only finitely many triangulations without non-trivial normal surfaces.

Actually, it turns out that such exceptional triangulations have a fixed number of tetrahedra, depending only on the $t$-invariant of the manifold. Despite this fact, there are plenty of triangulations without non-trivial normal surfaces: at least half of the $\sim 5000$ minimal triangulations of hyperbolic manifolds with $\leqslant 4$ tetrahedra are so $[\mathbf{1}, \mathbf{3}]$, and the number of such triangulations with $n$ tetrahedra is bigger than $n^{c n}$, for some $c>0$ and all $n>0[\mathbf{2}]$. (The proofs of [2] actually show the same results for triangulations without non-trivial $k$-normal surfaces.)

As the following result shows, there is only one example with closed manifolds, and it is the minimal triangulation $T_{5,2}$ of the lens space $L_{5,2}$, having one vertex and one tetrahedron.

Theorem 3. Every triangulation of any closed manifold except $T_{5,2}$ contains a non-trivial normal surface.

REMARK 4. We actually prove in Section 3 a stronger version of Theorems 2 and 3: with the same exceptions, every triangulation contains a non-trivial normal surface which intersects every edge in at most 2 points.

The $t$-invariant is essential in the proof of both theorems. Since their construction [12] in 1992, Turaev-Viro invariants have been used essentially only as a concrete tool to quickly distinguish triangulated manifolds. This is the first "theoretical" application of these invariants to 3-dimensional topology known to the authors.

Triangulated lens spaces. Using similar techniques we count the number of normal surfaces of non-negative Euler characteristic in the conjecturally minimal triangulations $T_{p, q}$ of all lens spaces. Let $S(p, q)$ be the sum of all partial quotients in the expansion of $p / q$ as a regular continued fraction. The triangulation $T_{p, q}$ contains $S(p, q)-3$ tetrahedra, see Section 4.

Theorem 5. Let $(p, q)$ be coprime with $p \geqslant 4$.

1) $T_{p, q}$ contains no normal projective planes and no non-trivial normal spheres;

2) the number $\tau\left(T_{p, q}\right)$ of distinct (up to normal isotopy) normal tori in $T_{p, q}$ is

$$
\tau\left(T_{p, q}\right)= \begin{cases}S(p, q)-3 & \text { if } q=1, \\ S(p, q)-4 & \text { in all other cases }\end{cases}
$$


3) the number $\kappa\left(T_{p, q}\right)$ of distinct (up to normal isotopy) normal Klein bottles in $T_{p, q}$ is

$\kappa\left(T_{p, q}\right)=\left\{\begin{array}{l}1 \quad \text { if } p=4 n \text { and } q=2 n \pm 1 \text { for some positive integer } n, \\ 0 \quad \text { in all other cases. }\end{array}\right.$

This paper is organized as follows. Precise definitions of all the concepts introduce above are given in Section 1 . Theorem 1 is proved in Section 2, while Theorems 2 and 3 are proved in Section 3. Theorem 5 is proved in Section 4.

Acknowledgments. We warmly thank Sergei Matveev for some helpful discussions.

\section{Surfaces and triangulations}

We fix here the terminology used in the Introduction and in the rest of the paper.

Let $T$ be a simplicial face-pairing of some tetrahedra, and $|T|$ its support. Here, $T$ is a triangulation of a closed 3-manifold $M$ when $M=|T|$. Tetrahedra are then not necessarily embedded in $M$. If $M$ has boundary, we say that $T$ is a triangulation of $M$ if $M$ is $|T|$ minus open stars of all its vertices (the latter is usually an ideal triangulation in the literature: here we suppress the word "ideal" for simplicity).

Let now $T$ be a triangulation of some compact 3-manifold $M$. We say that a closed embedded surface $\Sigma \subset M$ is $k$-normal to $T$ if the following holds:

- $\Sigma$ is transverse to $T$ and disjoint from $\partial M$;

- $\Sigma$ intersects each tetrahedron in discs, called normal discs;

- $\Sigma$ intersects each triangular face in arcs connecting distinct edges;

- each normal disc intersects each edge of the tetrahedron in at most $k$ points.

In a 1-normal surface, normal discs are triangles and squares as in Fig. 1(1), while in a 2-normal surface they can also be octagons as in Fig. 1-(2). Normal discs can be polygons with arbitrarily many edges as $k$ grows, as suggested by Fig. 1-(3).

A normal surface is then just a 1-normal surface. A trivial normal surface is one made only of triangles. Depending on whether the manifold is closed or not, a connected trivial normal surface is either the link of a vertex or parallel to the boundary.

Two $k$-normal surfaces in a triangulation $T$, intersecting each edge of $T$ in the same number of points are easily related via an isotopy, called normal isotopy.

A triangulation of a 3-manifold is minimal if the manifold cannot be triangulated with fewer tetrahedra. 


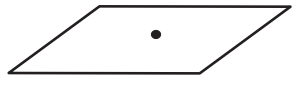

(1)

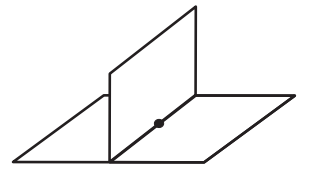

(2)

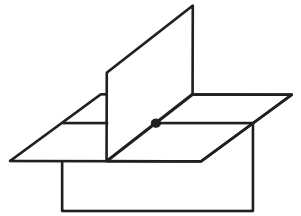

(3)

Figure 2. Some neighborhoods of points in an almost simple polyhedron.

We say that a compact 3-manifold $M$ with boundary is hyperbolic if it admits (after removing all tori from $\partial M$ ) a complete hyperbolic metric of finite volume (possibly with cusps and geodesic boundary). By Thurston's Hyperbolicity Theorem for Haken manifolds, this is equivalent to ask that every component of $\partial M$ has $\chi \leqslant 0$, and $M$ does not contain essential surfaces with $\chi \geqslant 0$.

\section{Complexity}

2.1. Definition. The proof of Theorem 1 relies heavily on complexity theory. We survey here the definition and some of its properties. We start with the following.

Definition 6. A compact 2-dimensional polyhedron $P$ is almost simple if the link of every point in $P$ is contained in the graph $Q$.

Alternatively, $P$ is almost simple if it is locally contained in the polyhedron shown in Fig. 2-(3). A point, a compact graph, a compact surface are therefore almost simple. Three important possible kinds of neighborhoods of points are shown in Fig. 2. A point having the whole of $\otimes$ as a link is called a vertex, and its regular neighborhood is shown in Fig. 2-(3). The set $V(P)$ of the vertices of $P$ consists of isolated points, so it is finite. Note that points, graphs, and surfaces do not contain vertices.

A compact polyhedron $P \subset M$ is a spine of a compact manifold $M$ with boundary if $M$ collapses onto $P$. When $M$ is closed, we say that $P \subset M$ is a spine of $M$ if $M \backslash P$ is an open ball.

Definition 7. The complexity $c(M)$ of a compact connected 3-manifold $M$ is the minimal number of vertices of an almost simple spine of $M$.

As an example, a point is a spine of $S^{3}$, and therefore $c\left(S^{3}\right)=0$. The complexity of a disconnected manifold is just defined as the sum of the complexities of its components.

2.2. Properties. We list here some properties of $c$, proved in [6], which will be used in the proof of Theorem 1. The first one is the following. 


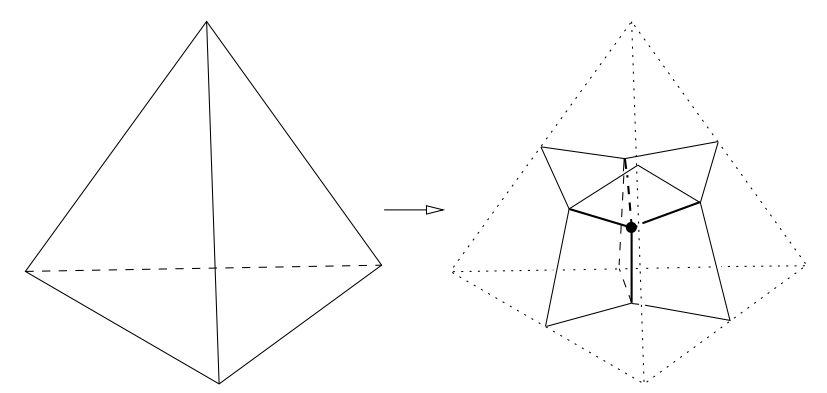

Figure 3. A spine of $M$ dual to a triangulation.

Theorem 8. Complexity is additive on connected sums. That is,

$$
c\left(M \# M^{\prime}\right)=c(M)+c\left(M^{\prime}\right)
$$

for all manifolds $M, M^{\prime}$.

As suggested by Fig. 3, a triangulation of a manifold $M$ with $n$ tetrahedra is dual to a polyhedron in $M$ with $n$ vertices. If $M$ is compact with boundary, the polyhedron is a spine of $M$. If it is closed, $M \backslash P$ consists of some $h$ open balls, and by removing open small discs from some appropriate $h-1$ faces of $P$ we get a spine of $M$. In both cases, we get a spine with at most $n$ vertices.

This shows that $c(M)$ is less or equal than the number of tetrahedra in a minimal triangulation of $M$. One key result in complexity theory, due to Matveev [6], says that the converse is true on the most interesting 3 -manifolds.

Theorem 9. Let $M$ be a closed irreducible or a bounded hyperbolic 3-manifold, different from $S^{3}, \mathbb{R P}^{3}$, and $L_{3,1}$. A minimal triangulation of $M$ contains precisely $c(M)$ tetrahedra.

2.3. From $\boldsymbol{k}$-normal surfaces to spines. If $\Sigma \subset M$ is a closed embedded surface in a compact 3-manifold $M$, we denote by $M_{\Sigma}$ the (possibly disconnected) compact manifold obtained from $M$ by removing an open tubular neighborhood of $\Sigma$. We have the following general result.

Proposition 10. Let $T$ be a triangulation of a manifold $M$ with $n$ tetrahedra, and let $\Sigma$ be a non-trivial $k$-normal surface. Then $c\left(M_{\Sigma}\right)<$ $n$.

Proof. We extend the construction shown in Fig. 3 to build a spine of $M_{\Sigma}$ with strictly less than $n$ vertices.

Let $\Delta$ be an abstract tetrahedron of the triangulation. The surface $\Sigma$ intersects $\Delta$ in discs, and each face of $\Delta$ into arcs connecting distinct edges. For each such arc, draw another parallel dashed arc closer to the nearest vertex, as in Fig. 4-(1). Also draw a central $Y$-shaped 

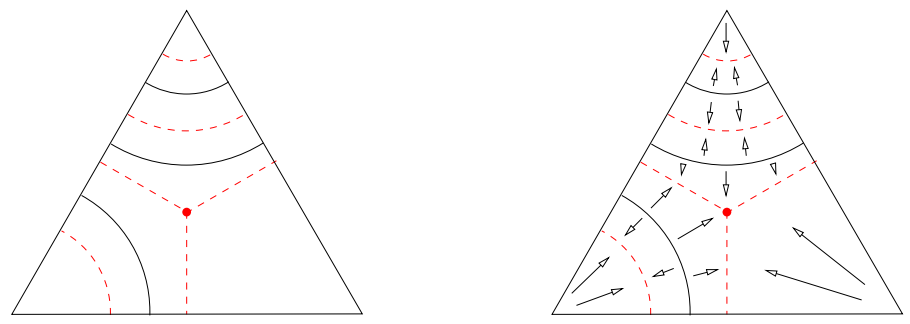

(1)

Figure 4. How to construct the graph $X(1)$, and a retraction onto it (2).

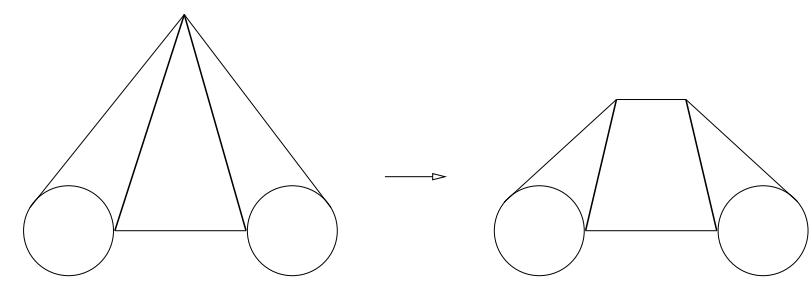

Figure 5. A perturbation of a cone into an almost simple polyhedron.

dashed graph as in the figure. Do this on each triangular face, so that the dashed arcs and graphs match along their endpoints. The resulting dashed object is a (possibly disconnected) graph $X$ in $\partial \Delta$ with 4 trivalent vertices.

The complement of $\Sigma$ in $\Delta$ consists of some topological balls. The boundary of each such abstract ball $B$ consists of some discs, arising from $\Sigma$, and one connected component $\partial_{v} B$ coming from $\partial \Delta$, which contains a connected component $X_{0}$ of the graph $X$. As shown in Fig. 4(2), $X_{0}$ is a spine of $\partial_{v} B \backslash$ vertices of $\left.\Delta\right\}$. We can extend the collapse to a collapse of $B$ onto the subpolyhedron $c\left(X_{0}\right) \subset B$ obtained by coning $X_{0}$ inside $B$.

We can force all dashed graphs and all collapses to match along the edges and faces of the triangulation. As a result, the union $P$ of all cones $c\left(X_{0}\right)$ is a spine of $M$. The polyhedron $P$ is made of points as in Fig. 2- $(1,2)$, except possibly at the vertices of the cones. Each $X_{0}$ is one of the following graphs: $\bigcirc, \oplus, \oslash$, and $\bigcirc-\bigcirc$. The cones on the first three graphs yield the pieces shown in Fig. 2. The cone on the fourth graph is not admitted in an almost simple spine; however, it can be locally perturbed to an almost simple object as shown in Fig. 5 .

Therefore $P$ is (after a suitable perturbation) an almost simple spine of $M$. Each $\otimes$ produces one vertex of $P$, while the other graphs yield no vertices. There can be at most one such graph for each $\Delta$ : this 
gives $c\left(M_{\Sigma}\right) \leqslant n$. Equality holds if and only if there is one $\otimes$ for each

tetrahedron. This happens precisely when $\Sigma$ intersects each tetrahedron into triangular faces, i.e. when $\Sigma$ is trivial.

q.e.d.

2.4. Proof of Theorem 1. Let $M$ be closed irreducible or bounded hyperbolic. A minimal triangulation $T$ contains precisely $c(M)$ tetrahedra, by Theorem 9. If $\Sigma$ is a non-trivial $k$-normal sphere, Proposition 10 gives $c\left(M_{\Sigma}\right)<c(M)$. But $M$ is irreducible, and hence $M_{\Sigma}=$ $M \# B^{3} \sqcup B^{3}$; since $c\left(B^{3}\right)=0$ and $c$ is additive on connected sums, we get $c\left(M_{\Sigma}\right)=c(M)$ : a contradiction. Theorem 1 is then proved.

\section{Constructions of normal surfaces}

3.1. Simple and special polyhedra. To prove Theorems 2 and 3 we need to introduce some definitions.

A compact 2-dimensional polyhedron is simple if the link of any point is homeomorphic to either $\bigcirc, \Phi$, or $\otimes$. That is, its regular neighborhood is one of those shown in Fig. 2. The points of type (1), (2), and (3) form a stratification of $P$ into vertices, 1-components and 2-components. The 1-stratum is the singular set $S(P)$, consisting of all points of type (2) and (3). If 1-components are indeed open segments and 2-components are open discs, they are called edges and faces, and the spine $P$ is special.

As shown above in Fig. 3, a triangulation $T$ is dual to a special polyhedron $P$. When $M$ has boundary, $P$ is a spine of $M$. If $M$ is closed, $P$ is a spine of $M$ minus some open balls.

3.2. Subpolyhedra and normal surfaces. Let $P$ be a special spine. Assign a colour - say, blue - to some faces of $P$. Let $Q$ be the union of the (closed) blue faces. It is easy to see that:

- $Q$ is a simple polyhedron if and only if the number of blue faces incident to each edge of $P$ is either 0,2 , or 3 , with multiplicity;

- $Q$ is a surface if and only if such a number is either 0 or 2 .

Moreover, it is also easy to see that every simple subpolyhedron arises in this way. This also shows that closed surfaces in $P$ are in natural bijection with $H_{2}\left(P, \mathbb{Z}_{2}\right)$.

Let $P$ be dual to a triangulation $T$. The following facts are noted in $[8]$ :

- if $Q$ is a surface, then it is normal in $T$;

- if $Q$ is a simple polyhedron, the boundary of a small regular neighborhood of $Q$ is a normal surface in $T$.

A normal surface arising in this way is said to be respectively of type $I$ and II. In fact, if a vertex of $P$ belongs to $Q$, then 4 possible configurations may arise, as shown in Fig. 6: the same figure shows how each configuration gives rise to normal triangles and squares. 


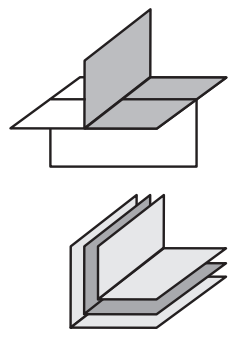

(1)

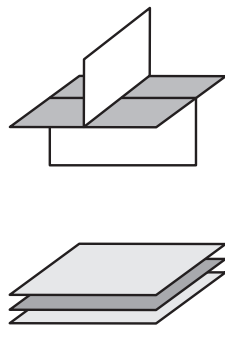

(2)

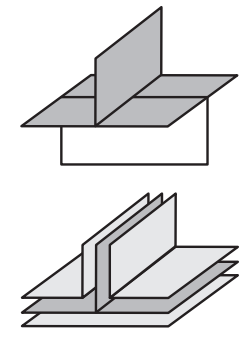

(3)

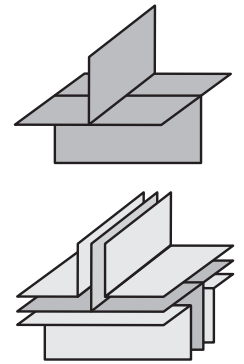

(4)

Figure 6. Four possible configurations of a simple subpolyhedron $Q$ near a vertex of $P$. The faces belonging to $Q$ are coloured. The boundary of a regular neighborhood consists respectively of (1) two triangles (2) two squares (3) two triangles and one square (4) four triangles.

3.3. Universal subpolyhedron. Let $T$ be a triangulation of a connected manifold $M$ with $k>0$ boundary components, and let $P$ be the dual spine. Then $M \backslash P$ consists of $k$ connected components. Assign a colour - say, blue - to those faces of $P$ which belong to the closure of two distinct components of $M \backslash P$. It is easy to see that the union $\Omega$ of the (closed) blue faces is a simple subpolyhedron of $P$. The following proposition shows that if $T$ does not contain non-trivial normal surfaces of type I or II then $\Omega$ is in some sense universal.

Proposition 11. Suppose $T$ does not contain any non-trivial normal surface of type $I$ or II. Then $\Omega$ is a surface with $k-1$ connected components, each parallel to some component of $\partial M$. Moreover, every proper simple subpolyhedron of $P$ is a subsurface of $\Omega$.

Proof. Suppose $P$ contains a proper simple subpolyhedron $Q$. As we said above, it induces a normal surface of type I or II, which must hence be trivial. Fig. 6 shows that configurations (2) and (3) give rise to squares, and are thus ruled out. If one vertex of $P$ is as in (4), all vertices of $P$ are so, because $P$ is connected: in this case $Q=P$. We are therefore left only with vertices of type (1); in particular, $Q$ is a surface.

The union $S_{1} \cup S_{2}$ of two surfaces in $P$ is a simple subpolyhedron, which is therefore either a surface or the whole of $P$. The second case is easily ruled out since both $S_{1}$ and $S_{2}$ look as in Fig. 6-(1) near the vertices. The subpolyhedron $\Omega$ is in fact the union of $k$ surfaces, each consisting of all faces separating one fixed boundary component to all others. Therefore $\Omega$ is itself a surface (and not the whole of $P$ ). 
Finally, every other proper simple subpolyhedron $Q$ is a trivial (i.e. boundary-parallel) surface, and hence contained in $\Omega$. It is also easy to conclude that $\Omega$ has $k-1$ components.

q.e.d.

There are plenty of triangulations $T$ that satisfy the hypothesis of Proposition 11. As shown in [2], this holds for instance when $\partial M$ consists of a genus- $g$ surface and $h$ tori, and $T$ has $g+h$ tetrahedra. The number of such examples with $n$ tetrahedra is bigger than $n^{c n}$ for some $c>0$ and all $n$, and the simplest one with at least two boundary components has 3 vertices [2].

3.4. Turaev-Viro invariants. One of the simplest invariants of Turaev-Viro type is the $t$-invariant defined by Matveev, Ovchinnikov, and Sokolov in [9]. It equals the homology-free 5-th Turaev-Viro invariant, and is defined as follows. Let $\varepsilon$ be a fixed root of the polynomial $x^{2}-$ $x-1$. Let $P$ be a simple spine of a manifold $M$ with boundary. Then

$$
t(P)=\sum_{Q \subset P}(-1)^{v_{Q}} \varepsilon^{\chi(Q)-v_{Q}}
$$

where the sum is taken over all simple subpolyhedra $Q$ of $P$, and $v_{Q}$ is the number of vertices of $Q$. It turns out that this quantity does not depend on $P$ and gives therefore a well-defined invariant $t(M)$ of $M$. If $M$ is a closed manifold, we define $t(M)$ as $t(M \backslash B)$ where $B$ is a small open ball.

Like all (suitably normalized) Turaev-Viro invariants, $t$ is multiplicative on connected sums (of closed manifolds) and $\partial$-connected sums (of manifolds with boundary).

3.5. Proof of Theorem 2. Let $M$ be a manifold with $k \geqslant 1$ boundary components, and $T$ be a triangulation of $M$ with $n$ tetrahedra. Let $P$ be its dual spine. We prove that, with finitely many exceptions, $T$ contains a normal surface of type I or II.

If not, by Proposition 11 there is a boundary-parallel surface $\Omega \subset P$ containing all proper simple subpolyhedra. Therefore

$$
t(M)=t(P)=(-1)^{n} \varepsilon^{\chi(M)-n}+t(\Omega) .
$$

Suppose there are infinitely many spines $P_{1}, \ldots, P_{i}, \ldots$ of $M$ without non-trivial normal surfaces. Each $P_{i}$ has $n_{i}$ vertices and contains some universal $\Omega_{i}$ as above.

The quantity $(-1)^{n_{i}} \varepsilon^{\chi(M)-n_{i}}+t\left(\Omega_{i}\right)$ must not depend on $i$. Since $\Omega_{i}$ is boundary-parallel, it may vary only in a finite set of topological types: hence $t\left(\Omega_{i}\right)$ varies in a finite set. On the other hand, the number $n_{i}$ of vertices of $P_{i}$ tends to $\infty$, since there are only finitely many special spines with a given number of vertices. Therefore $(-1)^{n_{i}} \varepsilon^{\chi(M)-n_{i}}$ takes infinitely many distinct values: a contradiction. 
3.6. Proof of Theorem 3. Let $T \neq T_{5,2}$ be a triangulation with $n$ tetrahedra of some closed 3-manifold $M$, and $P$ be the dual special spine. We prove that $T$ contains a non-trivial normal surface of type I or II.

If not, Proposition 11 implies that there is a surface $\Omega \subset P$ containing all proper simple subpolyhedra of $P$.

Suppose first that $T$ has $k \geqslant 2$ vertices. Therefore $P$ is a spine of $M$ minus $k$ small open balls. This manifold can be written as a $\partial$-connected sum

$$
(M \backslash B) \natural \underbrace{\left(S^{2} \times[0,1]\right) \natural \ldots \emptyset\left(S^{2} \times[0,1]\right)}_{k-1}
$$

where $B$ is a small open ball in $M$. By taking $S^{2}$ as a spine for $S^{2} \times[0,1]$ we see that $t\left(S^{2} \times[0,1]\right)=\varepsilon^{2}+1$. Since $t$ is multiplicative on $\partial$-connected sums, we have:

$$
t(P)=t(M \backslash B) \cdot t\left(S^{2} \times[0,1]\right)^{k-1}=t(M) \cdot\left(\varepsilon^{2}+1\right)^{k-1} .
$$

On the other hand, $\Omega$ consists of $k-1$ disjoint spheres, and therefore

$$
t(P)=(-1)^{n} \varepsilon^{\chi(P)-n}+t(\Omega)=(-1)^{n} \varepsilon^{k-n}+\left(\varepsilon^{2}+1\right)^{k-1} .
$$

The $t$-invariant is an element of the ring $\mathbb{Z}[\varepsilon]$. Since $k>1$, the equality

$$
t(M) \cdot\left(\varepsilon^{2}+1\right)^{k-1}=(-1)^{n} \varepsilon^{k-n}+\left(\varepsilon^{2}+1\right)^{k-1}
$$

implies that $\left(\varepsilon^{2}+1\right)$ divides $\varepsilon^{k-n}$. It is easy to see that $\varepsilon^{2}+1=\varepsilon+2$ is not invertible in $\mathbb{Z}[\varepsilon]$, while $\varepsilon^{i}$ is so for all $i$ : a contradiction.

Suppose now that $T$ has one vertex. Let $f$ be a face of $P$. By removing (the interior of) $f$ from $P$ we get an almost simple polyhedron $P^{\prime}$ such that $M \backslash P^{\prime}$ is an open solid torus. It collapses to a polyhedron $P^{\prime \prime} \subset P^{\prime}$ which is the union of a simple polyhedron $Q$ and a 1-dimensional graph. (Every almost simple spine collapses to a subpolyhedron of this kind, called nuclear by Matveev in [7].)

Since $Q$ is a proper subpolyhedron of $P$, it is empty by our assumption. Hence $P^{\prime \prime}$ is a graph, and since $M \backslash P^{\prime \prime}$ is an open solid torus, the manifold $M$ is a lens space. It is then shown in [7, Theorem 8.1.28] that $t(M) \in\left\{0,1, \varepsilon+1, \varepsilon^{2}+1\right\}$. Since $P$ contains no proper subpolyhedron, we have $t(P)=(-1)^{n} \varepsilon^{1-n}+1$, which belongs to $\left\{0,1, \varepsilon+1, \varepsilon^{2}+1\right\}$ only if $n=1$ and $t(P)=0$.

Finally, a direct investigation shows that there is a unique spine with $n=1$ vertices of a lens space with $t=0$. The lens space is $L_{5,2}$ and the triangulation is $T_{5,2}$, which indeed does not contain any non-trivial normal surface by Theorem 13 below, proved in [8].

Corollary 12. Every special spine of a closed manifold except the minimal spine of $L_{5,2}$ contains a proper simple polyhedron. 


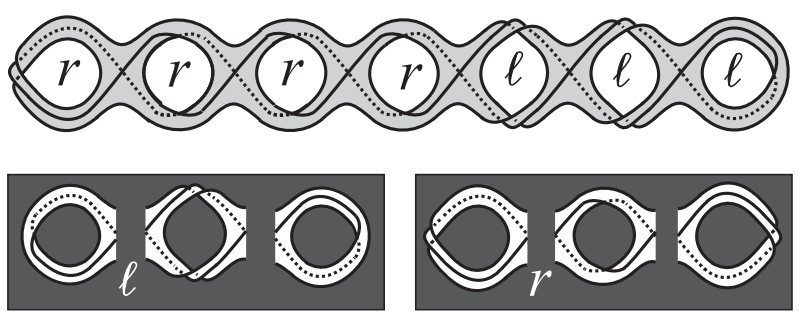

Figure 7. How to construct a spine of a lens space.

\section{Normal surfaces with nonnegative Euler characteristics in lens spaces}

4.1. The triangulations $\boldsymbol{T}_{\boldsymbol{p}, \boldsymbol{q}}$. Every lens space $L_{p, q}$ has a candidate minimal triangulation $T_{p, q}$, which is proved to be minimal only for finitely many values of $(p, q)$, and conjectured to be so in all cases. We compute here the number of normal surfaces of non-negative Euler characteristic in each such triangulation.

We describe the triangulation $T_{p, q}$ by drawing regular neighborhoods of the 1-skeleton of the dual spine: see [7] for details. Let $(p, q)$ be two positive coprime number, with $p \geqslant 4$. We define the operators $r, \ell: Z \oplus Z \rightarrow Z \oplus Z$ via the rules $r(a, b)=(a, a+b)$ and $\ell(a, b)=$ $(a+b, b)$. The pair $(q, p-q)$ can be obtained from the pair $(1,1)$ via a unique sequence $w$ of operators $r, \ell$. We use the string $w$ to construct the spine dual to $T_{p, q}$ as suggested in Fig. 7-down. For example, if

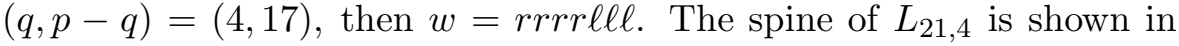
Fig. 7-up.

The following result was proved by Matveev and Fominykh in [8].

Theorem 13. Let $(p, q)$ be coprime with $p \geqslant 4$. Every connected normal surface in $T_{p, q}$ is either of type I or of type II.

Let $S(p, q)$ be the sum of all partial quotients in the expansion of $p / q$ as a regular continued fraction. The string $w$ contains $S(p, q)-2$ letters and thus $T_{p, q}$ contains $S(p, q)-3$ tetrahedra.

4.2. Proof of Theorem 5. Let $P$ be the special spine dual to $T_{p, q}$. By Theorem 13, every normal surface in $T_{p, q}$ is determined by some simple subpolyhedron of $P$. Let us analyze such polyhedra. One can easily see that every simple subpolyhedron $G$ of $P$ contains the singular graph $S(P)$ of $P$. This implies that $G$ can be obtained from $P$ by removing some faces of $P$. Denote by $s$ the number of removed faces. Since the Euler characteristic $\chi(P)$ of $P$ is equal to 1 , we have $\chi(G)=1-s$. Now we can conclude that $T_{p, q}$ does not contain any normal projective plane and any non-trivial normal sphere. Indeed, the boundary $F_{G}$ of a small regular neighborhood of $G$, i.e. a normal surface of type II, is orientable and its Euler characteristic is equal to $2-2 s$. Therefore, $F_{G}$ is a sphere 
if and only if $G=P$. But this sphere is, obviously, trivial. Finally, since $P$ is not a surface, the Euler characteristic of every normal surface of type I is less than 1.

As we know from [7], $P$ has $S(p, q)-3$ vertices and $S(p, q)-2$ faces. Note that there are at most two faces $f_{1}, f_{2}$ of $P$ that are incident more than once to some edge $e$. It can be checked directly that $f_{1}=f_{2}$ if and only if the sequence $w$ determining $P$ consists of operators $r$ only, i.e. if $q=1$. Since every simple subpolyhedron $G$ of $P$ contains the singular graph of $P, G$ contains both $f_{1}$ and $f_{2}$. Therefore, every normal surface of type I is nonorientable.

Let us prove the second assertion. As we already know, every normal torus in $T_{p, q}$ is a surface of type II, i.e. it is the boundary of a small regular neighborhood of some simple polyhedron $G$. Moreover, $G$ is obtained from $P$ by removing a face $f \neq f_{1}, f_{2}$ of $P$. If $p=5$ and $q=2$, then $P$ contains no proper simple subpolyhedra and hence $\tau\left(T_{5,2}\right)=0$. If $q=1$, then $f_{1}=f_{2}$ and we have $S(p, q)-3$ possibilities to choose $f$. For all other pairs $(p, q)$ we have $f_{1} \neq f_{2}$ and hence $\tau\left(T_{p, q}\right)=S(p, q)-4$.

Now let $F$ be a normal Klein bottle. Then $F$ is a surface of type I. Moreover, $F=P \backslash f$ for some face $f$ of $P$. Since $F$ contains the singular graph of $P, f$ is the unique face of $P$ incident once to each edge of $P$. Analyzing $P$ we can directly see that such a face $f$ exists if and only if the sequence $w$ determining $P$ is

$$
w=r \underbrace{l \ldots l}_{n-1} r
$$

for some positive integer $n$. Finally, we have

$$
(2 n-1,2 n+1)=r \underbrace{\ell \ldots \ell}_{n-1} r(1,1)
$$

and hence $p=4 n, q=2 n-1$.

Note that the conclusion (3) of Theorem 5 agrees with the results of Rubinstein [10], since any Klein bottle embedded in a lens space is incompressible and hence isotopic to a normal surface.

\section{References}

[1] R. Frigerio, B. Martelli \& C. Petronio, Complexity and Heegaard genus of an infinite class of compact 3-manifolds, Pacific J. Math. 210(2) (2003) 283297, MR 1988535, Zbl 1061.57017.

[2] R. Frigerio, B. Martelli \& C. Petronio, Dehn filling of cusped hyperbolic 3-manifolds with geodesic boundary, J. Differential Geom. 64(3) (2003) 425-455, MR 2032111, Zbl 1073.57010.

[3] R. Frigerio, B. Martelli \& C. Petronio, Small hyperbolic 3-manifolds with geodesic boundary, Experiment. Math. 13(2) (2004) 171-184, MR 2068891, Zbl 1068.57012. 
[4] C. Frohman \& J. Kania-Bartoszynska, The quantum content of the normal surfaces in a three-manifold, J. Knot Theory Ramifications 17(8) (2008) 10051033, MR 2439773, Zbl pre05363857.

[5] W. Jaco \& J. H. Rubinstein, 0-efficient triangulations of 3-manifolds, J. Differential Geom. 65(1) (2003) 61-168, MR 2057531, Zbl 1068.57023.

[6] S. V. Matveev, Complexity theory of three-dimensional manifolds, Acta Appl. Math. 19(2) (1990) 101-130, MR 1074221, Zbl 0724.57012.

[7] S. V. Matveev, Algorithmic topology and classification of 3-manifolds, Algorithms and Computation in Mathematics, Vol. 9, Springer-Verlag, Berlin, 2003, MR 1997069, Zbl 1048.57001.

[8] S. V. Matveev \& E. A. Fominykh, Normal surfaces in 3-manifolds, (Russian) Dokl. Akad. Nauk 384(6) (2002) 727-730; translation in Doklady Math. 65 (2002) 429-432, MR 1931107, Zbl pre05115787.

[9] S. V. Matveev, M. A. Ovchinnikov \& M. V. Sokolov, Construction and properties of the T-invariant, (Russian) Zap. Nauchn. Sem. S.-Peterburg. Otdel. Mat. Inst. Steklov. 267 (2000) 207-219; translation in J. Math. Sci. 113(6) (2003) 849-855. MR 1809828, Zbl 1033.57008.

[10] J. H. Rubinstein, On 3-manifolds that have finite fundamental group and contain Klein bottles, Trans. Amer. Math. Soc. 251 (1979) 129-137, MR 0531972, Zbl 0414.57005 .

[11] A. Thompson, Thin position and the recognition problem for $S^{3}$, Math. Res. Lett. 1(5) (1994) 613-630, MR 1295555, Zbl 0849.57009.

[12] V. G. Turaev \& O. Viro, State sum invariants of 3-manifolds and quantum 6j-symbols, Topology 31(4) (1992) 865-902, MR 1191386, Zbl 0779.57009.

Institute OF MATHEMATiCS AND MeChaniCS

URAL BRANCH OF THE

Russian ACADEMy of Sciences

16, S. Kovalevskaja Street 620219, EkAterinburg, Russia

E-mail address: fominykh@csu.ru

Dipartimento di Matematica UnIVERsità DI PISA Largo Pontecorvo 5 56127 Pisa, ItAly

E-mail address: martelli@dm.unipi.it 